\title{
Investigating Motivational Factors that Influence Football Match Attendance in the South African Premier Soccer League Post the 2010 FIFA World Cup
}

\author{
Dr Mlenga G. Jere \\ Graduate School of Business, University of Cape Town, \\ Private Bag X3, Rondebosch 7701 \\ Email: mlenga.jere@gsb.uct.ac.za
}

\author{
Mr Sundu Mathidza \\ University of Cape Town, Graduate School of Business, University of Cape Town \\ Private Bag X3, Rondebosch 7701 \\ Email: sundu@live.co.za
}

\section{Doi:10.5901/mjss.2014.v5n20p563}

\begin{abstract}
South Africa has a strong sporting culture and has successfully hosted big international sport events including the rugby, cricket, and soccer world cups. Spectators are an important factor in the success of such events. Using social exchange theory as the theoretical basis and employing motivational factors drawn from the literature on sport motivation, this study investigates the motivational factors that influence match attendance in the South African Premier Soccer League post the 2010 FIFA World Cup. The survey-based study employed a 26-item data collection instrument and a convenience sample of 70 respondents. Factor analysis was used to identifying six underlying motivational factors that influence match attendance. The findings show that the top three a priori motivational factors were entertainment, family appeal, and socialisation. These were supported by the factors extracted in the present study namely soccer subculture, family appeal, excitement seeking supporters, brand leisure seekers, entertainment, and vicarious achievers.
\end{abstract}

Keywords: Sport, motivational factors, South Africa, Premier Soccer League, football

\section{Introduction}

South Africa has a strong sporting culture and has successfully hosted big international sport events such as the rugby (1995), cricket (2003) and soccer (2010) world cups. The importance of spectators at sporting events and the impact of sporting events on the economy and various stakeholders have been acknowledged in previous research (Kruger, Botha \& Saayman, 2012). For soccer, the hosting of the 2010 FIFA World Cup was seen as an event that would galvanize South African soccer and impact on attendance at stadiums. Average attendance at football games has steadily decreased since the late 1990s. Except for games involving a few top teams, games are played to less than full stadiums. Given that hosting sport events is an expensive endeavour, falling attendance figures at stadiums translate into low ticket sales income and by implication poor sponsorship income. Ticket sales at football matches are not only an important direct source of income for clubs but also an indirect source of income such as sponsorships and broadcasting rights. Understanding what motivates people to attend soccer matches is therefore important for marketers to design appropriate events that will appeal to fans and attract them to attend matches (McDonald, Milne \& Hong, 2002; Snelgrove, Taks, Chalip \& Green, 2008). It is therefore the purpose of this paper to identify the motivational factors that influence football match attendance in the South African Premier Soccer League post the 2010 FIFA World Cup.

Social exchange theory (SET) is used as the theoretical basis for this paper. The focus of SET is that in their interactions, individuals expect and seek profit by their motivation to maximise rewards and minimise punishments. To realise their profit maximisation goals, individual design strategies that they belief would enhance their success. SET is therefore consistent with behavioural psychology in explaining behaviour as primarily driven by reward seeking and punishment avoidance. The substance of SET is that "social exchange comprises actions contingent on the rewarding reactions of others, which over time provide for mutually and rewarding transactions and relationships" (Cropanzano \& Mitchell, 2005, p890). SET is an important theory for explaining behaviour (Cropanzano \& Mitchell, 2005) according to which social exchange takes place when interactions between individuals happen and create obligations on the parties 
involved (Emerson, 1976). The interactions between individuals are interdependent and depend on the actions that the parties to the interaction take (Blau, 1964). If the interaction meets the needs of the parties involved, a positive relationship may result. Social exchange relationships that have beneficial consequences are more likely to engender the desired behaviour (Cropanzano \& Mitchell, 2005). The explanatory power of SET has been employed in the study of sports related subjects in diverse contexts (e.g., De Ruyter \& Wetzels, 2000; Kim, Gursoy \& Lee, 2006). Following below is a review of the literature on sport motivation factors, a description of the method used in the study, the study findings, and conclusion.

\section{Sport Motivational Factors}

Previous studies on sport motivational factors have identified many motivational factors. These include vicarious achievement (Sloan, 1989; Milne \& McDonald, 1999; Trail \& James, 2001; Funk et al., 2001; James \& Ross, 2004; Dubihlela, Dhurup \& Surujlal, 2009), skill mastery (Milne \& McDonald, 1999; James \& Ross, 2004; Dubihlela et al., 2009), family needs (Wann, 1995; Trail \& James, 2001; James \& Ross, 2004), socialisation (Wann, 1995; Milne \& McDonald, 1999; Trail \& James, 2001; Funk et al., 2009; James \& Ross, 2004; Dubihlela et al., 2009), diversion (Wann 1995; Trail \& James, 2001; Funk et al., 2009; James \& Ross, 2004; Dubihlela et al., 2009), and self-esteem (Wann, 1995; Milne \& McDonald, 1999; Funk et al., 2009). The other motivational factors are drama (Wann, 1995; Trail \& James, 2001; Funk et al., 2001; James \& Ross, 2004; Dubihlela et al., 2009), entertainment (Sloan, 1989; Wann, 1995; Funk et al., 2009; James \& Ross, 2004; Dubihlela et al., 2009), stress release (Sloan, 1989; Milne \& McDonald, 1999), performance, fan motivation, and identification with the sport subculture (Schouten \& McAlexander, 1995). Dubihlela and his colleagues' (2009) study which was undertaken in South Africa prior to the 2010 FIFA World Cup notes that six motivational factors were important in influencing fan decisions to attend PSL games namely social interaction, display of skill, escape, sharing in glory, enjoying the drama, and entertaining artistry (Dubihlela et al., 2009).

Some of these factors identified above namely socialisation, performance, esteem, diversion, fan motivation, leisure motivation, family appeal, entertainment and identification with a subculture are now discussed in detail. Because some motivational factors are closely related, an attempt is made to identify these and other terms used to refer to them in the literature. Sport consumers may attend events because of the opportunity for interpersonal relations and to be sociable. In the context of sport events, socialization has to do with interactions people have with others including family, friends, and other spectators. If an event is perceived as presenting better opportunities for socialisation, it is likely to be considered more favourably by potential attendees (Sloan, 1989; Funk et al., 2009). Socialisation is widely acknowledged as a fundamental motive for sport consumption (Irwin et al., 2008; Devasagayam \& Buff, 2008) and is closely related to the desire for group affiliation, camaraderie, and group identity (Funk et al., 2004; Armstrong, 2008). Some sport consumers attend sport events because they seek to experience the performance it provides (Funk et al., 2009). Performance refers to the perceived excellence, beauty, and creativity of the sport. According to Funk $(2004,2009)$, performance includes motives such as aesthetics, physical skill, aggression, and performance evaluation.

Esteem is the extent to which attendance to an event is perceived as providing an opportunity for vicarious achievement. For example, consumers who support a winning team bask in the success of the team and experience a sense of both personal and collective achievement. Other motives closely related to esteem include self-esteem, group affiliation, achievement, community support, and vicarious achievement (Funk et al., 2004; Funk et al., 2009). Another factor that influences event attendance is diversion. Diversion involves attending a sport event to escape the hassles and routine of one's everyday life. Some event consumers are motivated to attend sport events for the opportunity to escape and remove themselves from their daily work and life routines. Diversion is sought for its positive impact on mental wellbeing. Closely related to this factor are escape and fantasy (Funk et al., 2009).

Just as values are known to influence behaviour, fan behaviour and attitudes are driven by fan motives. Fan motivation relates to what motivates fanship, i.e., what motivate people to be fans (Wann et al., 2004). The literature identifies a number of scales for measuring fan motivation (e.g., Mahony et al., 2000, Zhang et al., 2001) that employ different variables but assume that motives influence fan behaviour and attitudes and that the higher one's fan motivation, the more likely they are to consume the sport of interest to them (Snelgrove et al., 2008). Typically, the sport is one of the possible leisure options under the consumer's consideration. The choice to attend, i.e., the leisure motivation, is based on the consumer's leisure preferences with consumers choosing activities that best meet their selection criteria. The leisure option's potential to provide learning, socialisation, and/or escape is known to influence event attendance (Kim \& Chalip, 2004).

Different sports have different subcultures associated with them. New members of these subcultures are socialised into these subcultures and begin to be identified with the subculture by others and themselves. Once socialised into the 
subculture, members adopt new beliefs, attitudes, and values which are a manifestation of their new culture (Snelgrove et al., 2008). The new culture and related values serve as guiding principles in the life of new member and the subculture (Rokeach, 1973; Schwartz, 1994) and direct attention and motivate behaviour (Schwartz, 1996). Identification with a subculture can have significant effects on sport consumption choices and preferences (Schouten \& McAlexander, 1995). But socialisation also takes place in the family. Family members are socialization agents for sport attitudes, beliefs, and consumption (Mullin, Hardy \& Sutton, 2000). Sport attendance decisions may therefore be influenced by family values, motivations, needs, bonding and the desire to share experiences with other family members (Armstrong, 2008), hence the importance of family appeal in sport marketing.

Finally, a sport event's entertainment value is also known to be an important factor in influencing sport attendance decisions (Zhang et al., 2004; Wann et al., 2004). Ferreira and Armstrong (2008) found that factors such as pre-game and in-game entertainment (such as the band, music, and pre-game activities) were important event attributes that impacted on sport attendance decisions.

The literature shows that different terms may be used to refer to the same motivational factors. It also acknowledges that motivational factors may be context specific. Coupled with Burgess \& Steenkamp's (2006) counsel for more research to be undertaken in emerging markets due to their idiosyncrasies compared to highly developed countries, it is fair to conclude that this study will contribute to the discourse on sport motivation factors. Further, it is not evident that the motivational factors identified by Dubihlela and colleagues (2009) before the 2010 FIFA World Cup would still apply due to the impact of the games on attitudes towards football. The purpose of this study is therefore to identify the motivational factors that influence football match attendance by putting together a survey instrument using factors culled from the literature.

\section{Method}

\subsection{Data collection}

Data collection employed a 26-item survey instrument of 5-point Likert-scale type of questions which respondents completed either online or at soccer matches. Respondents were asked to indicate whether the factors in the survey instrument were the reason for their attending or not attending matches ranging from 1=Strongly disagree to 5 Strongly agree. Convenience sampling was employed whereby people were randomly asked to complete the survey at soccer matches and on an online survey. Seventy usable responses were returned and included in the analysis.

\subsection{Data analysis}

Before performing factor analysis using IBM SPSS Statistics 21, the data was assessed for suitability. An inspection of the correlation matrix showed a number of correlations equal to and greater than 0.3 as required for factor analysis. The data was also considered appropriate for factor analysis because the Bartlett test of sphericity (Bartlett 1954) was significant $(p<0.05)$ and the Kaiser-Meyer-Olkin measure of sampling adequacy at .724 was greater that the recommended value of 0.6 (Kaiser, 1974).

To assess the nomological validity of the coding instrument, simple correlations were employed. According to Cohen (1988), effect sizes may be defined as either small ( $r=.10)$, medium ( $r=.30)$, or large $(r=.50)$. An examination of the correlation coefficients reveals that $83.7 \%$ of the intercorrelations are large, medium or small. This is compatible with convergent validity, that is, it provides support that the items in the coding instrument assess related concepts. Only $16.3 \%$ of the correlations had trivial effect sizes $(r<.10)$. To the extent that the variables that one expects to be related to each other exhibit some correspondence, the results provide support for convergent validity and discriminant validity and evidence for nomological (i.e., construct) validity. Principal components analysis was performed on the 26 items in the data collection instrument. Employing Kaiser's criterion of including components with eigenvalues greater than 1, 6 components qualified for extraction (Table 1) and were subjected to varimax rotation.

\section{Findings}

\subsection{Descriptive statistics}

The descriptive statistics show that the respondents agreed with all the 26 items used to measure the 9 motives that were included in this study. On the 5-point Likert scale (ranging from 1=Strongly disagree to 5 Strongly agree), the three items 
that were accorded the strongest affirmation were DIV2 (I attend PSL matches because I enjoy soccer) ( $M=4.03$, $\mathrm{SD}=1.09), \mathrm{FAN3}$ (I attend PSL matches because of my love for soccer) $(\mathrm{M}=4.02, \mathrm{SD}=1.09)$, and $\mathrm{SOC2}$ (PSL matches offer me the opportunity to socialise with friends) $(M=3.77, S D=1.29)$. The strong affirmation accorded to DIV2 suggests that the game itself appears to be a most important motive for attending soccer matches. Therefore, the quality of soccer needs to be maintained or even improved upon in order to increase the number of spectators who use soccer as a diversion to escape from the hassle of work and life routines.

The second item in terms of the strength of affirmation received was FAN3 (I attend PSL matches because of my love for soccer) ( $M=4.02, S D=1.09)$. This item is similar to DIV2 as it specifically identifies the game as the motive for attendance at soccer games. Fanship or fan motivation impacts on consumption behaviours such as attendance and attitudes to sport (Snelgrove et al., 2008). To grow this fanship, soccer marketers need to work on ensuring that those who love soccer are offered the opportunity to enjoy good games and also identify with the game. The third highest rated item was SOC2 (PSL matches offer me an opportunity to socialisation with my friends) ( $M=3.77, S D=1.29)$. It shows that there is there is general agreement that PSL matches offer consumers the opportunity to socialise with friends in addition to the enjoyment of soccer as reflected above. As shown by the means in Table 2, though to varying degrees, there was general agreement with the items in the survey instrument (with the exception of six items that had means below 2.5 on the 5-point scales).

Of the 9 a priori factors that were included in the survey (namely performance, esteem, diversion, family appeal, social interactions, entertainment, identification with subculture, leisure motivation, and fan motivation), the respondents gave the greatest affirmation to entertainment $(M=3.59)$, family appeal $(M=3.57)$, and socialisation $(M=3.35)$. The respondents' perception of PSL matches is that they offer exciting entertainment; they are packaged to appeal to the family, and are an opportunity for socialisation.

\subsection{Motivational factors}

The varimax rotation revealed a number of strong loadings (greater than 0.4 ) and very few cross loadings where items strongly loaded on more than one component (Table 2). The six factors that were extracted are labelled and described here. Accounting for $20.1 \%$ of the total variance explained, the first component has 3 high loading (>.8) marker variables namely CUL1 (I attend PSL matches because I enjoy soccer culture), FAN3 (I attend PSL matches because of my love for soccer), and DIV2 (I attend PSL matches because I enjoy soccer) and has been labelled soccer subculture. Led by CUL1, this component includes respondents who attend matches because they enjoy the soccer subculture, love soccer, and also enjoy soccer as a form of diversion. For this group, other important motives for attending soccer matches include socialisation with friends, relaxation (escape or diversion), the atmosphere at soccer stadiums, and leisure activity enjoyment. The second factor, labelled family appeal, accounts for $13.7 \%$ of the variance explained and has 4 high loading family related variables namely FAM1 (PSL matches are suitable for me to bring my family), FAM2 (PSL matches offer a safe environment for children), FAM3 (I attend PSL matches with my family), and SOC1 (PSL matches offer me an opportunity to socialise with my family). For consumers represented by this factor, the family appeal is what is what attracts them to stadiums, and they are generally agreed that PSL matches are suitable for families and offer a safe environment for children. Consequently, there was also agreement that PSL matches allow consumers to socialise with their families (SOC1).

The third factor labelled excitement seeking supporters is anchored by the variable FAN2 (I attend PSL matches because I belong to a supporters group or branch) suggesting that it's composed of soccer consumers who attend matches primarily because of their involvement with a supporters club. For this group, the standard of performance PER2 (The standard of football at PSL matches is good) is important because they acknowledge the standard of performance at PSL matches is good and that the matches excite them PER3 (PSL matches excite me). That they support a PSL team FAN1 (I attend PSL matches because I support a PSL team) is also an important motive for match attendance in this factor that accounts for $11.3 \%$ of the variance explained. The factor analysis also identified a brand leisure seekers group which accounts for $9 \%$ of the variance explained. For brand leisure seekers, the PSL brand is a major draw card as they attend matches mainly because they identify with and admire the PSL brand CUL3 (I attend PSL matches because the PSL brand is one that I admire), though they also identify soccer as their first choice leisure activity LEI2 (Attending PSL matches is my first choice leisure activity). They also attend matches because they reckon their ambitions and those of the teams they support are similar EST3 (I attend my team's PSL matches because their ambitions match mine) and also because the matches allow them to escape the hassles of life DIV1 (I attend PSL matches to escape the hassles of everyday normal life). 
Table 1: Factor analysis of motivational factor items (without poor loading items)

\begin{tabular}{|c|c|c|c|c|c|c|c|c|}
\hline \multirow{2}{*}{ Motivation variables (items) } & \multirow[b]{2}{*}{ Mean } & \multirow[b]{2}{*}{ SD } & \multicolumn{6}{|c|}{ Factors and factor loadings } \\
\hline & & & 1 & 2 & 3 & 4 & 5 & 6 \\
\hline CUL1 (I attend PSL matches because I enjoy soccer culture) & 3.65 & 1.10 & .831 & & & & & \\
\hline FAN3 (I attend PSL matches because of my love for soccer) & 4.02 & 1.09 & .811 & & & & & \\
\hline DIV2 (I attend PSL matches because I enjoy soccer) & 4.03 & 1.09 & .808 & & & & & \\
\hline SOC2 (PSL matches offer me an opportunity to socialisation with my friends) & 3.77 & 1.29 & .694 & & & & & \\
\hline DIV3 (Attending PSL matches allows me to relax) & 3.27 & 1.22 & .626 & & & .482 & & \\
\hline CUL2 (I attend PSL matches because of the atmosphere at the stadium) & 3.72 & 1.20 & .619 & & & & & .400 \\
\hline LEI1 (I attend PSL matches as it is a leisure activity I enjoy) & 3.48 & 1.07 & .612 & & & .456 & & \\
\hline SOC3 (PSL matches offer me an opportunity to socialisation with other fans) & 3.58 & 1.12 & .590 & & .414 & & & \\
\hline EST1 (I attend PSL matches to support my local team) & 3.56 & 1.25 & .529 & & .467 & & & \\
\hline FAM1 (PSL matches are suitable for me to bring my family) & 2.75 & 1.19 & & .901 & & & & \\
\hline FAM2 (PSL matches offer a safe environment for children) & 2.57 & 1.14 & & .829 & & & & \\
\hline FAM3 (I attend PSL matches with my family) & 2.40 & 1.21 & & .809 & & & & \\
\hline SOC1 (PSL matches offer me an opportunity to socialise with my family) & 2.69 & 1.21 & & .668 & & & & \\
\hline FAN2 (I attend PSL matches because I belong to a supporters group or branch) & 2.16 & 1.06 & & & .826 & & & \\
\hline PER2 (The standard of football at PSL matches is good) & 2.82 & 1.04 & & & .640 & & & \\
\hline PER3 (PSL matches excite me) & 3.06 & 1.16 & .487 & & .561 & & & \\
\hline FAN1 (I attend PSL matches because I support a PSL team) & 3.47 & 1.20 & .405 & & .526 & & & .489 \\
\hline CUL3 (I attend PSL matches because the PSL brand is one that I admire) & 2.25 & .93 & & & & 695 & & \\
\hline LEI2 (Attending PSL matches is my first choice leisure activity) & 2.20 & 1.09 & & & .491 & .571 & & \\
\hline EST3 (I attend my team's PSL matches because their ambitions match mine) & 2.48 & 1.10 & & & & .465 & & .419 \\
\hline DIV1 (I attend PSL matches to escape the hassles of everyday normal life) & 2.35 & 1.07 & & & & .437 & & \\
\hline ENT1 (PSL matches offer pre-game entertainment) & 2.43 & .96 & & & & & .832 & \\
\hline ENT2 GAME (PSL matches offer in-game entertainment) & 2.76 & 1.03 & & & & & .828 & \\
\hline ENT3 (There is enough entertainment offered at PSL matches) & 2.57 & .96 & & & & & .795 & \\
\hline EST2 (I attend PSL matches because of my team's reputation) & 2.81 & 1.13 & & & & & & .795 \\
\hline PER1 (My team's performance determines whether I attend a PSL match) & 2.83 & 1.31 & & & & & & .777 \\
\hline Eigenvalues & & & 9.68 & 2.59 & 2.20 & 1.59 & 1.39 & 1.16 \\
\hline$\%$ of variance & & & 37.24 & 9.96 & 8.47 & 6.10 & 5.36 & 4.46 \\
\hline Cumulative $\%$ of variance & & & 37.24 & 47.20 & 55.67 & 61.77 & 67.13 & 71.59 \\
\hline
\end{tabular}

The fifth factor is labelled entertainment and accounts for $9 \%$ of the variance. This factor includes consumers that appear to be drawn to matches primarily because of the pre-game ENT1 (PSL matches offer pre-game entertainment) and ingame ENT2 (PSL matches offer in-game entertainment) entertainment value they offer. The vicarious achievers factor is the last one and also accounts for $9 \%$ of the variance. For this group, match attendance is attributable to two motives: the reputation of the team EST2 (I attend PSL matches because of my team's reputation) and the team's performance PER1 (My team's performance determines whether I attend a PSL match).

In summary, this study sought to determine the motivational factors that influence football match attendance using a survey instrument that had 9 a priori factors (namely socialisation, performance, esteem, diversion, fan motivation, leisure motivation, family appeal, entertainment and identification with a subculture). When factor analysis was performed, 4 of the a priori factors (i.e., socialisation, performance, diversion, and leisure motivation) disappeared from the list of motivational factors applicable in this context while 5 were retained albeit with different nuances introduced by either the shedding of items or acquisition of items employed to measure the a priori factors (See Table 2 for this reallocation of items to factors). The 5 factors that were retained (with the new labels in brackets, where applicable) are identification with a subculture (soccer subculture), family appeal, fan motivation (excitement seeking supporters), entertainment, and esteem (vicarious achievement). A new factor emerged namely brand leisure.

\section{Conclusion}

The purpose of this study was to identify the motivational factors that influence football match attendance. Based on the mean responses, the top three influencers of match attendance identified were DIV2 (I attend PSL matches because I enjoy soccer), FAN3 (I attend PSL matches because of my love for soccer), and SOC2 (PSL matches offer me an opportunity to socialisation with my friends). This suggests that in order to attract more spectators to attend football matches, consideration should be given to improving these dimensions of the soccer experience. Firstly, the quality of soccer must be good enough to be considered valuable and judged enjoyable by the spectators. The DIV2 (I attend PSL matches because I enjoy soccer), dimension relates mostly to the functional aspects of the soccer experience. The love 
people profess for soccer needs to be improved and sustained by improving the soccer experience and therefore emotional attachment associated with the soccer experience. As soccer is perceived as an opportunity for socialisation, deliberate effort needs to be directed at determining and enhancing important elements relating to the social dimension of the soccer experience as well.

The findings above are supported by the factors that emerged from factor analysis which extracted six factors. The top three of these were soccer subculture, family appeal, and excitement seeking supporters. The soccer subculture includes the variable DIV2 (I attend PSL matches because I enjoy soccer), affirming the importance of making soccer enjoyable. But there are other significant variables (such as socialisation, relaxation, esteem, and leisure) in this factor, implying that in practice the soccer experience is multifaceted and that spectators make emotional investments in the teams they support and vicariously share in the achievements and losses of the teams they support.

For some soccer fans, soccer is a family affair. The implication of this is the soccer experience must be suitable for the family (including being child friendly) and offer opportunities for family socialisation. In addition to family, spectators may also below to a community of supporters whose main interests include excitement and socialisation. These factors also require consideration from practitioners in designing the soccer experience. The last three factors that also require consideration relate to the PSL brand, additional entertainment, and local team reputation and performance.

In closing, the contribution of this study must be viewed in light of its major weakness that we hope future research will address namely the small sample. Even though the data were assessed and found suitable for use in factor analysis, the use of small tends to increase sampling errors compared to large samples.

\section{References}

Armstrong, K.L. (2008). Consumers of color and the "culture" of sport attendance: Exploratory insights. Sport Marketing Quarterly, 17(4), 218-231.

Bartlett, M.S. (1954). A note on the multiplying factors for various chi square approximations. Journal of the Royal Statistical Society, 16 (Series B), 296-298.

Blau, P.M. (1964). Exchange and power in social life. New York: John Wiley.

Burgess, S.M., \& Steenkamp, J.-B.E. M. (2006). Marketing renaissance: research in emerging markets advances marketing science and practice. International Journal of Research in Marketing, 23(4), 337-356.

Cohen, J. (1988). Statistical power analysis for the behavioral sciences. Hillsdale, NJ: Erlbaum.

Cropanzano, R., \& Mitchell, M.S. (2005). Social exchange theory: An interdisciplinary review. Journal of Management, 31, 874-900.

De Ruyter, K., \& Wetzels, M. (2000). With a little help from my fans: Extending models of pro-social behaviour to explain supporters behaviour to buy soccer club shares. Journal of Economic Psychology, 21, 387-409.

Dubihlela, J., Dhurup, M., \& Surujlal, J. (2010). Dimensions of attachment among fans within the South African premier soccer league (PSL) and relationship with attendance. African Journal for Physical, Health Education, Recreation and Dance, 16(3), 491-509.

Emerson, R.M. (1976). Social exchange theory. Annual Review of Sociology, 2, 335-362.

Funk, D.C., \& James, J. (2001). The psychological continuum model: A conceptual framework for understanding an individual's psychological connection to sport. Sport Management Review, 2, 119-150.

Funk, D.C., Filo, K., Beaton, A.A., \& Pritchard, M. (2009). Measuring the motives of sport event attendance: Bridging the academicpractitioner divide to understanding behaviour. Sport Marketing Quarterly, 18(3), 126-138.

Funk, D.C., Ridinger, L.L., \& Moorman, A.M. (2004). Exploring origins of involvement: Understanding the relationship between consumer motives and involvement with professional sport teams. Leisure Science, 26, 35-62.

James, J., \& Ross, S. (2002). The motives of sport consumers: A comparison of Major and Minor League baseball. International Journal of Sport Management, 3(3), 180-198.

James, J.D., \& Ross, S.D. (2004). Comparing sport consumer motivations across multiple sports. Journal of Sport Behavior, 5(3), 17-25.

Kaiser, H. (1974). An index of factorial simplicity. Psychometrika, 39, 31-36.

Kim, N-S., \& Chalip, L. (2004). Why travel to the FIFA World Cup? Tourism Management, 25, 695-707.

Kim, H.J., Gursoy, D., \& Lee, S-B. (2006). The impact of the 2002 World Cup on South Korea: Comparisons of pre- and post-games. Tourism Management, 27(1), 86-96.

Kruger, M., Botha, K., \& Saayman, M. (2012). The relationship between visitor spending and repeat purchase visits: An analysis of spectators at the Old Mutual Two Oceans Marathon. Acta Commercii, 12(1), 108-122.

Mahony, D.F., Madrigal, R., \& Howard, D.R. (2000). Using the psychological commitment to team (PCT) scale to segment sport consumers based on loyalty. Sport Marketing Quarterly, 9(1), 15-25.

McDonald, D.R. (2000). Does Bat Day make cents? The effect of promotions of the demand for Major League Baseball. Journal of Sport Management, 1(14), 8-27.

McDonald, M.A., Milne, R.G., \& Hong, J. (2002). Motivational factors for evaluating sport spectator and participant markets. Sport Marketing Quarterly, 11(2), 100-113.

Milne, G.R., \& McDonald, M.A. (1999). Sport marketing: Managing the exchange process. Sudbury, MA: Jones \& Bartlett Publishers.

Mullin, B., Hardy, S., \& Sutton, W.S. (2000). Sport Marketing (2nd Ed). Champaign, IL: Human Kinetics. 
Rokeach, M. (1973). The nature of human values. New York: Free Press.

Schouten, J.W., \& McAlexander, J.H. (1995). Subcultures of consumption: An ethnography of the new bikers. Journal of Consumer Research, 22(1), 43-6.

Schwartz, S.H. (1994). Are there universal aspects in the structure and contents of human values? Journal of Social Issues, 50(4), 1945.

Schwartz, S.H. (1996). Value priorities and behavior: Applying of theory of integrated value systems. In C. Seligman, J. M. Olson \& M. P. Zanna (Eds.), The Psychology of Values: The Ontario Symposium (Vol. 8, pp. 1-24). Hillsdale, NJ: Erlbaum.

Sloan, L.R. (1989). The motives of sports fans. In J.D. Golldstein, Sports, Games, and Play: Social and Psychological Viewpoints (2nd Edition, pp 175-240). Hillsdale, NJ: Erlbaum Associates.

Snelgrove, R., Taks, M., Chalip, L., \& Green, B.C. (2008). How visitors and locals at a sport event differ in motives and identity. Journal of Sport \& Tourism, 13(3), 165-180.

Trail, G.T., \& James, J.D. (2001). The motivation scale for sport consumption: Assessment of the scale's psychometric properties. Journal of Sport Behavior, 24(1), 108-127.

Wann, D., Bayens, C., \& Driver, A. (2004). Likelihood of attending a sporting event as a function of ticket scarcity and team identification. Sport Marketing Quarterly, 13(4), 209-215.

Wann, D.L. (1995). Preliminary validation of the Sport Fan Motivation Scale. Journal of Sport and Social Issues, 19(4), $377-396$.

Zhang, J.J., Lam, E.T., Connaughton, D.P., Bennett, G., Pease, D.G., Pham, U.L., Killion, L.E., Ocker, L.B., \& Duley, A.R. (2004). Variables affecting spectator enjoyment of minor league hockey games. International Journal of Sport Management, 5(2), 157182. 